\title{
Of Sheep and Wolves: Curtailing Coverage for Essential Imaging Tests Based on Flawed Use and Cost Arguments
}

\author{
Johannes Czernin ${ }^{1}$, Martin Allen-Auerbach ${ }^{2,3}$, and Jeremie Calais ${ }^{2,3}$ \\ ${ }^{1}$ Editor-in-Chief, JNM; ${ }^{2}$ Ahmanson Translational Theranostics Division, David Geffen School of Medicine at UCLA, Los Angeles, \\ California; and ${ }^{3}$ Department of Molecular and Medical Pharmacology, David Geffen School of Medicine at UCLA, Los Angeles, \\ California
}

$\mathrm{I}$ In a recently published study ( 1 ) that compared ${ }^{18} \mathrm{~F}$-fluciclovine with ${ }^{68} \mathrm{Ga}$-PSMA 11 for detecting early biochemical prostate recurrence, Calais et al. provide a trial profile that includes a small, interesting, and most likely overlooked detail. One hundred fortythree patients were screened for eligibility, and 93 had to be excluded. Thirty-one of ninety-three patients did not meet inclusion criteria, and 43 of 93 declined to participate. However, in 19 of 93 patients insurance denied coverage of the ${ }^{18} \mathrm{~F}$-fluciclovine scan.

${ }^{18} \mathrm{~F}$-fluciclovine had received Food and Drug Administration approval on May 27, 2016. This was followed by a Centers for Medicare \& Medicaid Services (CMS) coverage decision on January 1,2017 . Yet, these decisions have not swayed Aetna to cover ${ }^{18}$ F-fluciclovine scans. Their most recent Policy \#0071 (2) concludes, "Aetna considers fluciclovine ${ }^{18} \mathrm{~F}$ PET experimental and investigational for prostate cancer and for all other indications because of insufficient evidence."

Blue Shield, on the other hand, has made some progress: their policy document from November 2018 (Blue Shield CA Medical Policy - 6.01.52 (3)) states that "PET scanning with ${ }^{11} \mathrm{C}$-choline and ${ }^{18} \mathrm{~F}$-fluciclovine may be medically necessary for evaluating suspected or biochemically recurrent prostate cancer after primary treatment to detect small-volume disease in soft tissues." Although the language ("may be considered") leaves much room for coverage denial, this still represents progress.

Consistent with the overall insurance culture, CMS in 2013 also created an unusual exception for PET imaging. It determined that "3 FDG PET scans are covered under $\S$ 1862(a)(1)(A) when used to guide subsequent management of antitumor treatment strategy after completion of initial anticancer therapy. Coverage of any additional FDG PET scans (i.e., beyond 3) used to guide subsequent management of antitumor treatment strategy after completion of initial antitumor therapy will be determined by local Medicare Administrative Contractors." Given the multiple lines of treatments available for many cancers, these arbitrary limits are anachronistic.

Importantly, successful cancer treatments are associated with precipitous decreases in tumor glucose utilization that are imaged accurately with ${ }^{18}$ F-FDG PET/CT (4). Conversely, if tumor glucose utilization does not decrease after a single treatment cycle, the therapy cannot and will not work (immuno-therapy approaches may occasionally represent an exception to this rule). Glucose metabolic tumor activity is thus a uniquely powerful intermediate endpoint biomarker that informs accurately about treatment failure early during treatment (5). Given the severe side effects and exorbitant costs of therapies (financial toxicity) (6), insurance companies

COPYRIGHT @ 2019 by the Society of Nuclear Medicine and Molecular Imaging. should promote rather than curtail the use of ${ }^{18} \mathrm{~F}-\mathrm{FDG} \mathrm{PET} /(\mathrm{CT})$. The perception that $\mathrm{PET} / \mathrm{CT}$ is expensive has been pervasive and debunked before (7). As PET/ CT imaging provides whole-body molecular and anatomic assessments, it is actually very inexpensive. It accounts for only a small portion of cancer care and cancer imaging costs (7).

A recent study published in JAMA (8) analyzed changes in use rates of $\mathrm{CT}$, MRI, ultrasound, and nuclear medicine

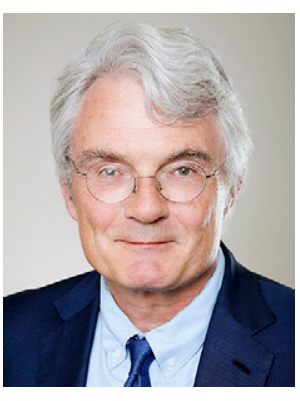

Johannes Czernin across all use sectors (not limited to cancer). CT use increased annually by around 3\% from 2010 to 2016. In contrast, nuclear medicine studies decreased significantly (by more than 20\%) in each age group and across various health plans. For example, CT use (for all indications not only cancer) increased from 204 per 1,000 person-years in 2000 to 428 per 1,000 person-years in 2016 whereas all nuclear medicine studies combined decreased from 94 to 64 per 1,000 person-years. Thus, CT use alone is around 7-fold higher than all nuclear medicine modalities together (including high-volume tests such as cardiac SPECT imaging). These findings underscore the low use of nuclear medicine tests and further suggest an alarmingly low use of PET in oncology (as PET is part of the nuclear medicine use analysis).

We thus have a toxic scenario: PET has the reputation of being expensive, an odd notion given that standard-of-care PET/CT studies include whole-body PET and diagnostic-quality CT studies. Nevertheless, this perception has led to further limits in ${ }^{18} \mathrm{~F}-\mathrm{FDG}$ PET use (lifetime cap for Medicare patients; tedious and frustrating preauthorization procedures for non-Medicare patients).

Aetna (9), Blue Cross/Blue Shield (10), and CMS (11) pride themselves in promoting precision medicine. Determining treatment responses, early, using ${ }^{18} \mathrm{~F}-\mathrm{FDG}$ PET without limitations, provides a simple precision oncology tool (12). Consistently covering Food and Drug Administration-approved diagnostic tests, such as fluciclovine PET, would go a long way toward improved patient care (precision oncology).

The nuclear medicine community has at times been very successful in turning the tide (National Oncologic PET Registry). Yet, we have not completely overcome the industry's Pavlovian resistance reflex against PET imaging. We have (reluctantly) accepted reimbursement cuts, limitations on clinical indications, vague coverage language, and evasive insurance behavior. We have tolerated endless peer-to-peer discussions with "experts" who are poorly informed about imaging technologies.

A recent study analyzed Organisation for Economic Co-operation and Development-derived health-care spending data across 11 


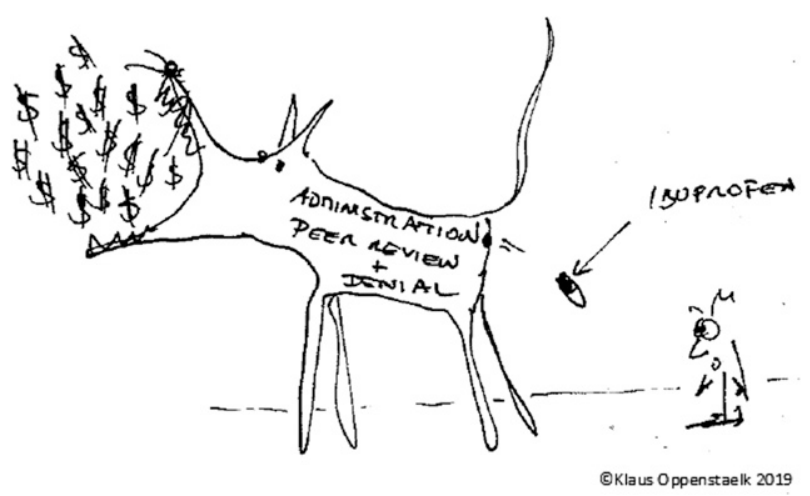

FIGURE. Courtesy of Klaus Oppenstaelk.

high-income countries (13). The United States spends $17.8 \%$ of its gross domestic product on health care, which is almost twice as much as the average of other developed countries. These expenses do not translate uniformly into better outcomes. The United States has the highest overweight and obesity levels, lowest life expectancy, and highest infant mortality. On a positive note, smoking rates were second lowest among the 11 countries. To achieve these mediocre outcomes, the U.S. health-care systems spend $8 \%$ of total expenses on governance and administration versus an average of $3 \%$ in the other 10 countries analyzed. It is thus not surprising that a large proportion of U.S. physicians are dissatisfied with the high administrative burden, paperwork, and time spent on insurance claims. Use of CT and MRI ranked number 1 and 2, among the countries, respectively. However, the authors concluded that "prices of labor and goods, including pharmaceuticals, and administrative costs appear to be the major drivers of the difference in overall cost between the United States and other high-income countries" (13).

Maybe insurances could focus more on "prices of labor and goods, including pharmaceuticals, and administrative costs" when attempting to decrease health-care spending. ${ }^{18} \mathrm{~F}-\mathrm{FDG}$ PET, ${ }^{68} \mathrm{Ga}-$ DOTATATE PET (14), and ${ }^{18} \mathrm{~F}-$ fluciclovin PET (15), as well as all nuclear medicine studies combined, are minor contributors to cancer care costs. These important imaging studies should be available without any barriers (as is the case for CT and MRI).

\section{REFERENCES}

1. Calais J, Ceci F, Eiber M, et al. ${ }^{18}$ F-fluciclovine PET-CT and ${ }^{68} \mathrm{Ga}-\mathrm{PSMA}-11$ PET-CT in patients with early biochemical recurrence after prostatectomy: a prospective, singlecentre, single-arm, comparative imaging trial. Lancet Oncol. 2019;20:1286-1294.

2. Aetna. Positron emission tomography (PET): number 0071. Aetna website. http://www. aetna.com/cpb/medical/data/1_99/00 Positron Emission Tomography (PET) 71.html. Accessed October 21, 2019.

3. Clinical guideline: PET imaging, any site. Magellan Healthcare website. http:// www.radmd.com/assets/Clinical\%20Guidelines//Health\%20Plan\%20Specific \% 20Guidelines\%202018/PET\%20Imaging\%20Any\%20Site\%20\%E2\%80\% 93\%20BSC\%202018\%20v3.pdf. Accessed October 21, 2019.

4. Wahl RL, Jacene H, Kasamon Y, Lodge MA. From RECIST to PERCIST: evolving considerations for PET response criteria in solid tumors. J Nucl Med. 2009;50:122S-150S

5. Weber WA. Assessing tumor response to therapy. J Nucl Med. 2009;50:1S-10S.

6. Carrera PM, Kantarjian HM, Blinder VS. The financial burden and distress of patients with cancer: Understanding and stepping-up action on the financial toxicity of cancer treatment. CA Cancer J Clin. 2018;68:153-165.

7. Yang Y, Czernin J. Contribution of imaging to cancer care costs. J Nucl Med. 2011;52:86S-92S

8. Smith-Bindman R, Kwan ML, Marlow EC, et al. Trends in use of medical imaging in US health care systems and in Ontario, Canada, 2000-2016. JAMA. 2019;322:843-856.

9. Aetna. Precision medicine. Aetna website. http://www.aetna.com/employer-plans/ document-library/aetna-precision-medicine-v1.pdf. Accessed October 21, 2019.

10. BlueCross BlueShield. Topic: precision medicine. BlueCross BlueShield website. https://www.bcbs.com/the-health-of-america/topics/precision-medicine. Accessed October 21, 2019.

11. CMS manual system. Centers for Medicare \& Medicaid Services website. https://www.cms.gov/Regulations-and-Guidance/Guidance/Transmittals/downloads/ R156NCD.pdf. Accessed November 14, 2019.

12. Czernin J, Sonni I, Razmaria A, Calais J. The future of nuclear medicine as an independent specialty. J Nucl Med. 2019;60:3S-12S.

13. Papanicolas I, Woskie LR, Jha AK. Health care spending in the United States and other high-income countries. JAMA. 2018;319:1024-1039.

14. Czernin J, Ceci F. Aetna and ${ }^{68}$ Ga-DOTATATE: a sequel to "the injustice of being judged by the errors of others." J Nucl Med. 2018;59:721-722.

15. Parent EE, Schuster DM. Update on ${ }^{18} \mathrm{~F}$-fluciclovine PET for prostate cancer imaging. J Nucl Med. 2018;59:733-739. 